\title{
Mass composition anisotropy with the Telescope Array Surface Detector data
}

\section{Yana Zhezher ${ }^{a, b, *}$ on behalf of the Telescope Array Collaboration}

(a complete list of authors can be found at the end of the proceedings)

\author{
a Institute for Cosmic Ray Research, University of Tokyo, \\ Kashiwanoha 5-1-5, Kashiwa, Chiba, Japan \\ ${ }^{b}$ Institute for Nuclear Research, Russian Academy of Sciences, \\ 60-letya Oktyabrya 7a, Moscow, Russia \\ E-mail: zhezher.yana@physics.msu.ru
}

Mass composition anisotropy is predicted by a number of theories describing sources of ultrahigh-energy cosmic rays. Event-by-event determination of a type of a primary cosmic-ray particle is impossible due to large shower-to-shower fluctuations, and the mass composition usually is obtained by averaging over some composition-sensitive observable determined independently for each extensive air shower (EAS) over a large number of events. In the present study we propose to employ the observable $\xi$ used in the TA mass composition analysis for the mass composition anisotropy analysis. The $\xi$ variable is determined with the use of Boosted Decision Trees (BDT) technique trained with the Monte-Carlo sets, and the $\xi$ value is assigned for each event, where $\xi=1$ corresponds to an event initiated by the primary iron nuclei and $\xi=-1$ corresponds to a proton event.

Use of $\xi$ distributions obtained for the Monte-Carlo sets allows us to separate proton and iron candidate events from a data set with some given accuracy and study its distributions over the observed part of the sky. Results for the TA SD 11-year data set mass composition anisotropy will be presented.

$3^{\text {th }}$ International Cosmic Ray Conference (ICRC 2021)

July 12 th - 23rd, 2021

Online - Berlin, Germany

\footnotetext{
${ }^{*}$ Presenter
} 


\section{Introduction}

Ultra-high-energy cosmic rays (UHECRs) are most energetic particles observed at the Earth coming from somewhere outside of our own Galaxy. They pose a number of theoretical questions, yet unanswered despite a century of experimental observations. The origin and acceleration mechanisms of UHECRs are two of those issues, which are still unknown at all energies.

The mass composition of the UHECRs at Earth is one of the measurable quantities directly connected to the cosmic-ray acceleration mechanisms in the source and source population. Mass composition is usually derived from the observed cascades of secondary particles initiated in the interactions of cosmic rays with the Earth's atmosphere, extensive air showers (EAS). It may be done via a measurement of the longitudinal shape of EAS with fluorescence telescopes. In this case, by reconstructing the depth of the shower maximum $\mathrm{X}_{\max }$, one may directly relate this observable to the mass composition of primary particles [1].

An alternative method was proposed by the Telescope Array collaboration from the analysis of solely the surface detector data, which has an important advantage of much higher statistics compared to the fluorescence measurements [2]. The former method is based on the multivariate analysis technique, namely the Boosted Decision Trees (BDT) which employs the set of 16 composition-sensitive observables reconstructed from the data. These observables are transformed into a single variable $\xi$ which then determines the average atomic mass of a primary particle $\langle\ln A\rangle$.

Going further, mass composition anisotropy is also predicted in a number of astrophysical models [3]. Different astrophysical scenarios, varying the UHECR source density as well as the injection composition and energy spectrum, lead to different distribution of primary particles as will be observed at Earth, if one takes into account the propagation of the UHECRs in the cosmological photon backgrounds and in the extragalactic and Galactic magnetic fields.

Due to the deflections of UHECRs in Galactic and extragalactic magnetic fields if one want to analyze the association of cosmic-ray events with possible sources, it makes it reasonable to consider only the highest energy cosmic rays, because their magnetic rigidity is expected to be greater (unless they also have a higher charge), and because the number of sources visible from Earth is much smaller, due to the GZK effect. On the other hand, one may also consider analyzing the distribution of proton events only, as both light and energetic particles will be affected in the least way during their propagation to Earth.

The methods described above use a large number of observed extensive air showers to derive the average $\langle\ln A\rangle$ as a function of energy, and due to large fluctuations in signals registered from EAS, the current level of experimental analysis techniques doesn't allow to determine the mass of a primary particle separately for each observed shower. In our study, we use the TA SD $\xi$ parameter as a mass indicator and analyze the distribution of high-energy proton events in the TA SD 11-year data set. As it was shown previously [4] with the Monte-Carlo simulations, $\xi$ parameter distributions are potentially sensitive to the UHECR mass composition anisotropies.

\section{Telescope Array experiment}

The Telescope Array (TA) experiment is the largest ultra-high-energy (UHE) cosmic-ray experiment in the Northern hemisphere, located near Delta, Utah, USA. TA is designed to register 
the extensive air showers (EAS) caused by the UHE cosmic rays entering the atmosphere. The experiment operates in hybrid mode and performs simultaneous measurements of the particle density and timing at the ground level with the surface detector array (SD) [5] and the fluorescence light with 38 fluorescence telescopes grouped into three fluorescence detector stations [6]. The SD is an array of 507 plastic scintillator detectors arranged on a square grid with $1.2 \mathrm{~km}$ spacing covering an area of approximately $700 \mathrm{~km}^{2}$. Each detector is composed of two layers of $1.2 \mathrm{~cm}$ thick extruded scintillator of the $3 \mathrm{~m}^{2}$ effective area.

\section{Data set and Monte-Carlo simulations}

We employ the 11-year data set from the TA surface detector, covering from 11th May 2008 up to 10th May 2019. After applying the quality cuts, the final dataset used for the analysis consists of 21628 events in the energy range from $10^{18} \mathrm{eV}$ to $10^{20} \mathrm{eV}$ with the following quality cuts applied both to the data and MC events:

1. Events with 7 or more triggered counters

2. Events with zenith angle $\theta<45^{\circ}$.

3. Events with reconstructed core position of at least $1200 \mathrm{~m}$ away from the edge of the array.

4. Events with $\chi_{G}^{2} /$ d.o.f. $<4$ and $\chi_{L D F}^{2} /$ d.o.f. $<4$ [7].

5. Events with arrival direction reconstructed with accuracy less than $5^{\circ}$.

6. Events with the fractional uncertainty of the $S_{800}$ less than $25 \%$ [7].

7. Events with $E>10^{18} \mathrm{eV}$.

For the Monte-Carlo simulations, CORSIKA software package is used together with the QGSJETII-03 model for high-energy hadronic interactions, FLUKA for low energy hadronic interaction and EGS4 for electromagnetic processes. The detector response is simulated with the use of the GEANT4 package. Real-time array status and detector calibration information for 9 years of observations are used for each simulated event [8]. Two separate Monte-Carlo data sets, for proton and iron primaries, are simulated and stored in the same data format as the SD data in the energy range $10^{17.5}-10^{20.5} \mathrm{eV}$.

\section{4. "Proton-like" event separation}

In the present mass composition anisotropy study we want to focus only on those of the events which we may consider to be of a proton nature. To separate the proton events from our data set, we use the distribution of $\xi$ parameter for proton and iron Monte-Carlo sets, and derive criterion $\xi_{c u t}(E)$, such as that data events with $\xi<\xi_{\text {cut }}$ may be considered to be proton events.

We define the statistical significance of "signal" events (protons) in the presence of "background" events (iron nuclei) as following: 

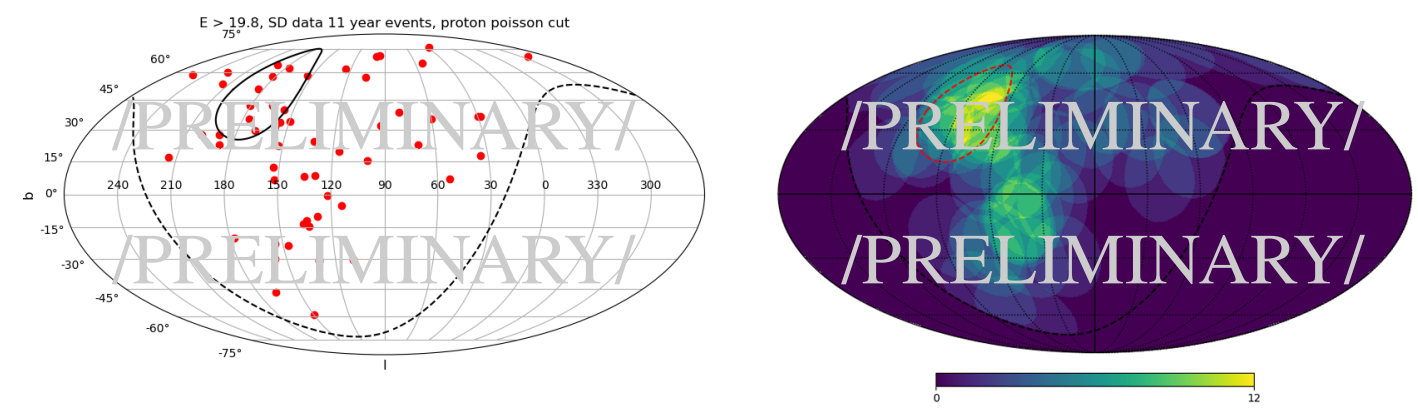

Figure 1: "Proton-like" event distribution in the experimental dataset with energies $\log (E / \mathrm{eV})>19.8$ : left - distribution of events (red dots), black line corresponds to the hotspot area; right $-20^{\circ}$ oversampling, red dashed line corresponds to the hostpot area.

$$
S=\frac{N_{\text {signal }}}{\sqrt{N_{\text {background }}}},
$$

where $N_{\text {signal }}$ and $N_{\text {background }}$ is the number of signal and background events, correspondingly. Then we find the $\xi$ values which maximize the significance in the MC set as a function of energy and use them consequently on our data events.

\section{Results and discussion}

In the Figure 1, we show the distribution of "proton-like" events derived from experimental data with energies greater than $10^{19.8} \mathrm{eV}$. On the left side, distribution of events is shown, where each event is represented with a red dot. On the right side we present a $20^{\circ}$ oversampling [9]. One can clearly see the excess of proton events in the hotspot area and another fainter one in the Galactic plane region. Both distributions are presented in Galactic coordinates.

In order to estimate the significance of the observed excess, we proceed with the following analyses. First of all, we evaluate the pre-trial significance of the excess as following:

- We generate the isotropic MC set with data composition obtained in [2] and much higher statistics, assuming it to be a mixture of $p$ and $F e$ events.

- "Proton-like" events are then selected from this MC set with the same cuts as from the experimental data.

- The MC "proton-like" event set is also oversampled, and Li-Ma significance is calculated at each pixel with the following equation:

$$
S_{\mathrm{LM}}=\sqrt{2}\left[N_{\text {on }} \ln \left(\frac{(1+\eta) N_{\text {on }}}{\eta\left(N_{\text {on }}+N_{\text {off }}\right)}\right)+N_{\text {off }} \ln \left(\frac{(1+\eta) N_{\text {off }}}{N_{\text {on }}+N_{\text {off }}}\right)\right]^{1 / 2}
$$

$N_{\text {on }}$ - number of "proton-like" events in the experimental dataset in the specific direction, $N_{\text {off }}$ - number of background "proton" events from the isotropic $\mathrm{MC}, \eta$ - ratio of a number of events in "on" and "off" datasets. 


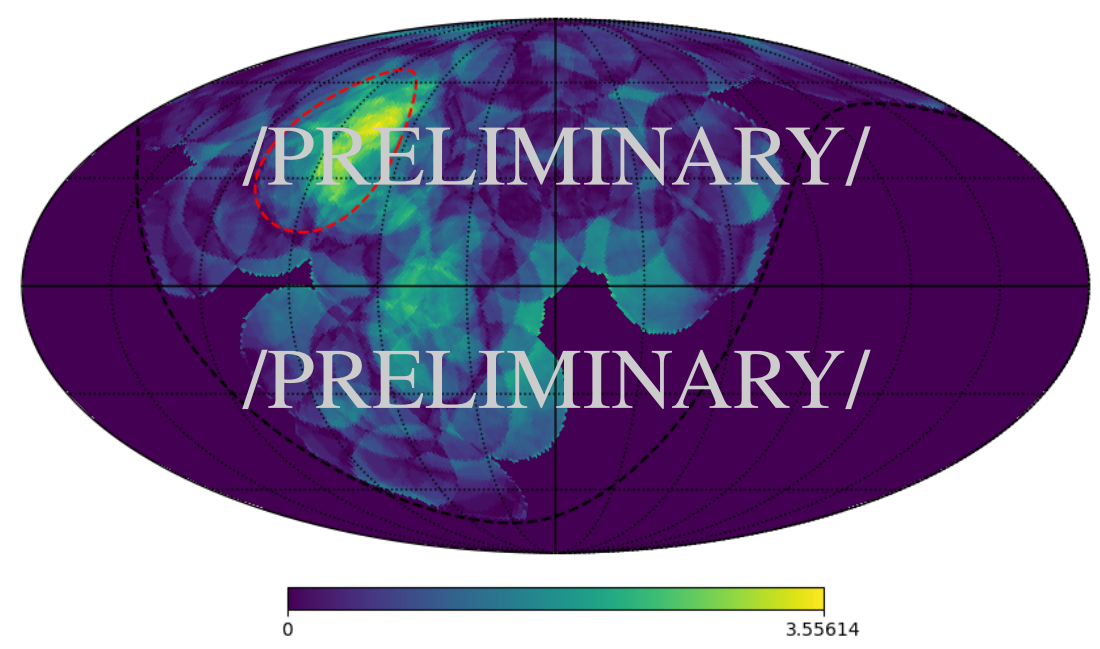

Figure 2: Distribution of the maximum Li-Ma significance for the "proton" excess. Red dashed line corresponds to the hotspot area.

The distribution of the maximum Li-Ma significance is shown in Figure 2, which show the pre-trial significance of the excess to be $3.56 \sigma$.

Next, we come to the calculation of a post-trial significance, which takes into account the sporadic occurrences of these kind of excesses in the data, even if there is no underlying anisotropy.

To calculate the post-trial significance, we create a large number of mock random "proton" data sets with energies of events $\log (E / e V)>19.8$ and calculate the pre-trial Li-Ma significance of each of these sets. After that, we count how often the $3.56 \sigma$ excesses occur in our mock datasets, and translate this frequency into the post-trial significance.

The distribution of Li-Ma significances for the mock "proton" datasets is shown in Figure 3, where the $3.56 \sigma$ significance is shown with vertical red line. The occurrence of such significance among 30000 generated datasets is 1369 , which corresponds to the post-trial significance of the observed proton excess of $1.7 \sigma$.

To summarize the result, no significant mass composition anisotropy is observed in the TA SD 11-year dataset. Given the efficiency of proton selection of about 50\%, the observed local significance in the hotspot region coincides with the local significance of the hotspot itself [10], given that events are partially selected from the dataset.

\section{Acknowledgments}

The Telescope Array experiment is supported by the Japan Society for the Promotion of Science(JSPS) through Grants-in-Aid for Priority Area 431, for Specially Promoted Research JP21000002, for Scientific Research (S) JP19104006, for Specially Promoted Research JP15H05693, for Scientific Research (S) JP15H05741 and JP19H05607, for Science Research (A) JP18H03705, for Young Scientists (A) JPH26707011, and for Fostering Joint International Research (B) JP19KK0074, by the joint research program of the Institute for Cosmic Ray Research (ICRR), The University 


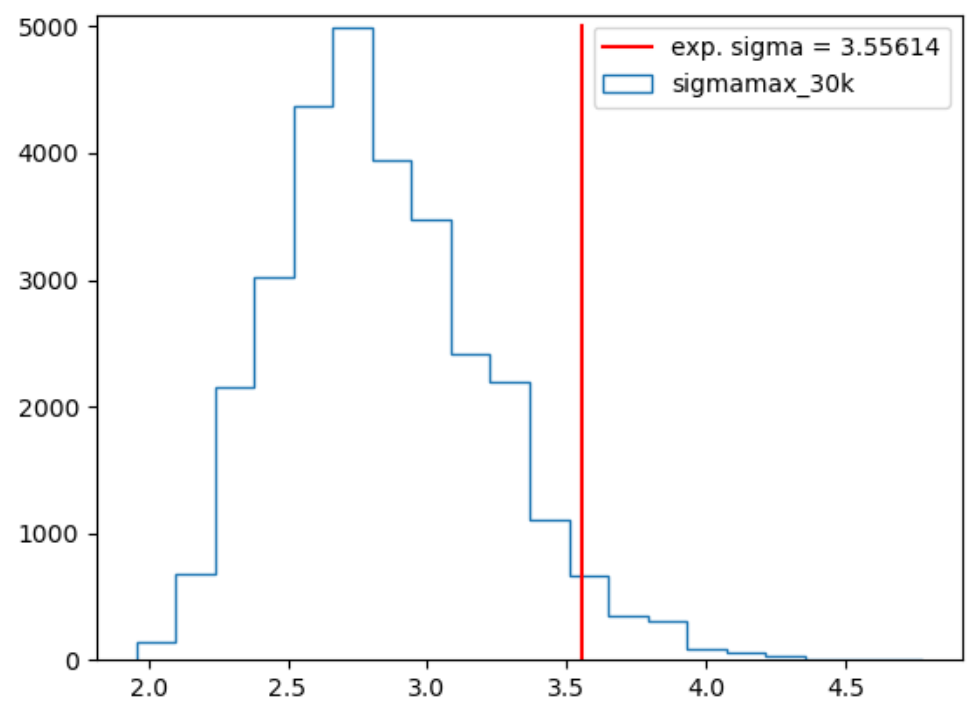

Figure 3: Distribution of Li-Ma significance for the mock "proton" event datasets. Pre-trial significance is denoted with vertical red line.

of Tokyo; by the Pioneering Program of RIKEN for the Evolution of Matter in the Universe (r-EMU); by the U.S. National Science Foundation awards PHY-1404495, PHY-1404502, PHY1607727, PHY-1712517, PHY-1806797 and PHY-2012934; by the National Research Foundation of Korea (2017K1A4A3015188, 2020R1A2C1008230, \& 2020R1A2C2102800) ; IISN project No. 4.4501.18, and Belgian Science Policy under IUAP VII/37 (ULB). The development and application of the machine learning analysis method is supported by the Russian Science Foundation grant No. 17-72-20291 (INR). This work was partially supported by the grants of the joint research program of the Institute for Space-Earth Environmental Research, Nagoya University and Inter-University Research Program of the Institute for Cosmic Ray Research of University of Tokyo. The foundations of Dr. Ezekiel R. and Edna Wattis Dumke, Willard L. Eccles, and George S. and Dolores Doré Eccles all helped with generous donations. The State of Utah supported the project through its Economic Development Board, and the University of Utah through the Office of the Vice President for Research. The experimental site became available through the cooperation of the Utah School and Institutional Trust Lands Administration (SITLA), U.S. Bureau of Land Management (BLM), and the U.S. Air Force. We appreciate the assistance of the State of Utah and Fillmore offices of the BLM in crafting the Plan of Development for the site. Patrick A. Shea assisted the collaboration with valuable advice and supported the collaboration's efforts. The people and the officials of Millard County, Utah have been a source of steadfast and warm support for our work which we greatly appreciate. We are indebted to the Millard County Road Department for their efforts to maintain and clear the roads which get us to our sites. We gratefully acknowledge the contribution from the technical staffs of our home institutions. An allocation of computer time from the Center for High Performance Computing at the University of Utah is gratefully acknowledged. 


\section{References}

[1] HiRes collaboration, T. K. Gaisser et al., Cosmic ray composition around 10**18-eV, Phys. Rev. D 47 (1993) 1919-1932.

[2] Telescope Array collaboration, R. U. Abbasi et al., Mass composition of ultrahigh-energy cosmic rays with the Telescope Array Surface Detector data, Phys. Rev. D 99 (2019) 022002, [1808.03680].

[3] B. R. d'Orfeuil, D. Allard, C. Lachaud, E. Parizot, C. Blaksley and S. Nagataki, Anisotropy expectations for ultra-high-energy cosmic rays with future high statistics experiments, Astron. Astrophys. 567 (2014) A81, [1401.1119].

[4] Y. Zhezher, G. Rubtsov, P. Sokolsky and S. Troitsky, Anisotropy in the mass composition from the Telescope Array Surface Detector data, PoS ICRC2019 (2020) 494.

[5] T. Abu-Zayyad, R. Aida, M. Allen, R. Anderson, R. Azuma, E. Barcikowski et al., The surface detector array of the Telescope Array experiment, Nuclear Instruments and Methods in Physics Research A 689 (Oct., 2012) 87-97, [1201 . 4964].

[6] H. Tokuno, Y. Tameda, M. Takeda, K. Kadota, D. Ikeda, M. Chikawa et al., New air fluorescence detectors employed in the Telescope Array experiment, Nuclear Instruments and Methods in Physics Research A 676 (June, 2012) 54-65, [1201. 0002].

[7] D. Ivanov, Energy spectrum measured by the telescope array surface detector, Ph.D. thesis, Rutgers The State University of New Jersey - New Brunswick, May, 2012.

[8] Telescope Array collaboration, T. Abu-Zayyad et al., CORSIKA Simulation of the Telescope Array Surface Detector, 1403.0644.

[9] N. Hayashida, M. Nagano, D. Nishikawa, H. Ohoka, N. Sakaki, M. Sasaki et al., The anisotropy of cosmic ray arrival directions around $10^{18} \mathrm{eV}$, Astroparticle Physics 10 (May, 1999) 303-311, [astro-ph/9807045].

[10] Telescope Array collaboration, R. U. Abbasi et al., Indications of Intermediate-Scale Anisotropy of Cosmic Rays with Energy Greater Than 57 EeV in the Northern Sky Measured with the Surface Detector of the Telescope Array Experiment, Astrophys. J. Lett. 790 (2014) L21, [1404 . 5890]. 


\section{Full Authors List: Telescope Array Collaboration}

R.U. Abbasi ${ }^{1}$, M. Abe ${ }^{2}$, T. Abu-Zayyad ${ }^{1,3}$, M. Allen ${ }^{3}$, Y. Arai ${ }^{4}$, E. Barcikowski ${ }^{3}$, J.W. Belz ${ }^{3}$, D.R. Bergman ${ }^{3}$, S.A. Blake ${ }^{3}$, I. Buckland ${ }^{3}$, R. $\mathrm{Cady}^{3}$, B.G. Cheon ${ }^{5}$, J. Chiba ${ }^{6}$, M. Chikawa ${ }^{7}$, T. Fujii ${ }^{8}$, K. Fujisue ${ }^{7}$, K. Fujita ${ }^{4}$, R. Fujiwara ${ }^{4}$, M. Fukushima ${ }^{7,9}$, R. Fukushima ${ }^{4}$, G. Furlich ${ }^{3}$, R. Gonzalez ${ }^{3}$, W. Hanlon ${ }^{3}$, M. Hayashi ${ }^{10}$, N. Hayashida ${ }^{11}$, K. Hibino ${ }^{11}$, R. Higuchi ${ }^{7}$, K. Honda ${ }^{12}$, D. Ikeda ${ }^{11}$, T. Inadomi ${ }^{13}$, N. Inoue ${ }^{2}$, T. Ishii ${ }^{12}$, H. Ito ${ }^{14}$, D. Ivanov ${ }^{3}$, H. Iwakura ${ }^{13}$, H.M. Jeong ${ }^{15}$, S. Jeong ${ }^{15}$, C.C.H. Jui ${ }^{3}$, K. Kadota ${ }^{16}$, F. Kakimoto ${ }^{11}$, O. Kalashev ${ }^{17}$, K. Kasahara ${ }^{18}$, S. Kasami ${ }^{19}$, H. Kawai ${ }^{20}$, S. Kawakami ${ }^{4}$, S. Kawana ${ }^{2}$, K. Kawata ${ }^{7}$, E. Kido ${ }^{14}$, H.B. Kim ${ }^{5}$, J.H. Kim ${ }^{3}$, J.H. Kim ${ }^{3}$, M.H. Kim ${ }^{15}$, S.W. Kim ${ }^{15}$, Y. Kimura ${ }^{4}$, S. Kishigami ${ }^{4}$, Y. Kubota ${ }^{13}$, S. Kurisu ${ }^{13}$, V. Kuzmin ${ }^{17, * *}$, M. Kuznetsov ${ }^{17,21}$, Y.J. Kwon ${ }^{22}$, K.H. Lee ${ }^{15}$, B. Lubsandorzhiev ${ }^{17}$, J.P. Lundquist ${ }^{3,23}$, K. Machida ${ }^{12}$, H. Matsumiya ${ }^{4}$, T. Matsuyama ${ }^{4}$, J.N. Matthews ${ }^{3}$, R. Mayta ${ }^{4}$, M. Minamino ${ }^{4}$, K. Mukai $^{12}$, I. Myers ${ }^{3}$,

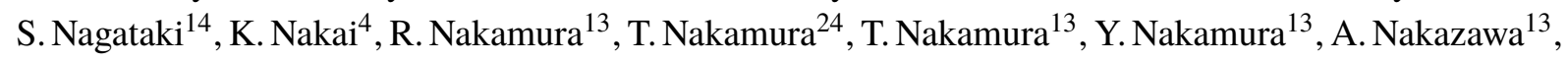
T. Nonaka ${ }^{7}$, H. Oda ${ }^{4}$, S. Ogio ${ }^{4,25}$, M. Ohnishi ${ }^{7}$, H. Ohoka ${ }^{7}$, Y. Oku ${ }^{19}$, T. Okuda ${ }^{26}$, Y. Omura ${ }^{4}$, M. Ono ${ }^{14}$, R. Onogi ${ }^{4}$, A. Oshima ${ }^{4}$, S. Ozawa ${ }^{27}$, I.H. Park ${ }^{15}$, M. Potts ${ }^{3}$, M.S. Pshirkov ${ }^{17,28}$, J. Remington ${ }^{3}$, D.C. Rodriguez ${ }^{3}$, G.I. Rubtsov ${ }^{17}$, D. Ryu ${ }^{29}$, H. Sagawa ${ }^{7}$, R. Sahara ${ }^{4}$, Y. Saito ${ }^{13}$, N. Sakaki ${ }^{7}$, T. Sako ${ }^{7}$, N. Sakurai ${ }^{4}$, K. Sano ${ }^{13}$, K. Sato ${ }^{4}$, T. Seki ${ }^{13}$, K. Sekino ${ }^{7}$, P.D. Shah ${ }^{3}$, Y. Shibasaki ${ }^{13}$, F. Shibata ${ }^{12}$, N. Shibata ${ }^{19}$, T. Shibata ${ }^{7}$, H. Shimodaira ${ }^{7}$, B.K. Shinn ${ }^{29}$, H.S. Shin ${ }^{7}$, D. Shinto ${ }^{19}$, J.D. Smith ${ }^{3}$, P. Sokolsky ${ }^{3}$, N. Sone ${ }^{13}$, B.T. Stokes ${ }^{3}$, T.A. Stroman ${ }^{3}$, T. Suzawa ${ }^{2}$, Y. Takagi ${ }^{4}$, Y. Takahashi ${ }^{4}$, M. Takamura ${ }^{6}$, M. Takeda ${ }^{7}$, R. Takeishi ${ }^{7}$, A. Taketa $^{30}$, M. Takita $^{7}$, Y. Tameda ${ }^{19}$, H. Tanaka ${ }^{4}$, K. Tanaka ${ }^{31}$, M. Tanaka ${ }^{32}$, Y. Tanoue ${ }^{4}$, S.B. Thomas ${ }^{3}$, G.B. Thomson ${ }^{3}$,

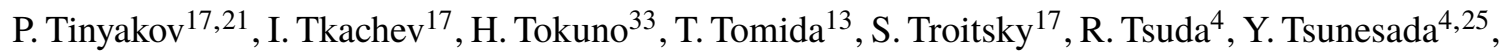

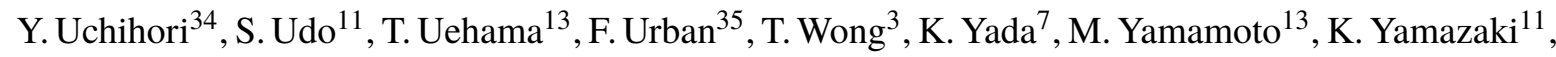
J. Yang ${ }^{36}$, K. Yashiro ${ }^{6}$, F. Yoshida ${ }^{19}$, Y. Yoshioka ${ }^{13}$, Y. Zhezher ${ }^{7,17}$, and Z. Zundel ${ }^{3}$

${ }^{1}$ Department of Physics, Loyola University Chicago, Chicago, Illinois, USA

${ }^{2}$ The Graduate School of Science and Engineering, Saitama University, Saitama, Saitama, Japan

${ }^{3}$ High Energy Astrophysics Institute and Department of Physics and Astronomy, University of Utah, Salt Lake City, Utah, USA

${ }^{4}$ Graduate School of Science, Osaka City University, Osaka, Osaka, Japan

5 Department of Physics and The Research Institute of Natural Science, Hanyang University, Seongdong-gu, Seoul, Korea

${ }^{6}$ Department of Physics, Tokyo University of Science, Noda, Chiba, Japan

${ }^{7}$ Institute for Cosmic Ray Research, University of Tokyo, Kashiwa, Chiba, Japan

8 The Hakubi Center for Advanced Research and Graduate School of Science, Kyoto University, KitashirakawaOiwakecho, Sakyo-ku, Kyoto, Japan

${ }^{9}$ Kavli Institute for the Physics and Mathematics of the Universe (WPI), Todai Institutes for Advanced Study, University of Tokyo, Kashiwa, Chiba, Japan

${ }^{10}$ Information Engineering Graduate School of Science and Technology, Shinshu University, Nagano, Nagano, Japan

${ }^{11}$ Faculty of Engineering, Kanagawa University, Yokohama, Kanagawa, Japan

${ }^{12}$ Interdisciplinary Graduate School of Medicine and Engineering, University of Yamanashi, Kofu, Yamanashi, Japan

${ }^{13}$ Academic Assembly School of Science and Technology Institute of Engineering, Shinshu University, Nagano, Nagano, Japan

${ }^{14}$ Astrophysical Big Bang Laboratory, RIKEN, Wako, Saitama, Japan

15 Department of Physics, Sungkyunkwan University, Jang-an-gu, Suwon, Korea

16 Department of Physics, Tokyo City University, Setagaya-ku, Tokyo, Japan

${ }^{17}$ Institute for Nuclear Research of the Russian Academy of Sciences, Moscow, Russia

${ }^{18}$ Faculty of Systems Engineering and Science, Shibaura Institute of Technology, Minato-ku, Tokyo, Japan 
${ }^{19}$ Department of Engineering Science, Faculty of Engineering, Osaka Electro-Communication University, Neyagawashi, Osaka, Japan

${ }^{20}$ Department of Physics, Chiba University, Chiba, Chiba, Japan

${ }^{21}$ Service de Physique Théorique, Université Libre de Bruxelles, Brussels, Belgium

${ }^{22}$ Department of Physics, Yonsei University, Seodaemun-gu, Seoul, Korea

23 Center for Astrophysics and Cosmology, University of Nova Gorica, Nova Gorica, Slovenia

${ }^{24}$ Faculty of Science, Kochi University, Kochi, Kochi, Japan

${ }^{25}$ Nambu Yoichiro Institute of Theoretical and Experimental Physics, Osaka City University, Osaka, Osaka, Japan

${ }^{26}$ Department of Physical Sciences, Ritsumeikan University, Kusatsu, Shiga, Japan

${ }^{27}$ Quantum ICT Advanced Development Center, National Institute for Information and Communications Technology, Koganei, Tokyo, Japan

${ }^{28}$ Sternberg Astronomical Institute, Moscow M.V. Lomonosov State University, Moscow, Russia

${ }^{29}$ Department of Physics, School of Natural Sciences, Ulsan National Institute of Science and Technology, UNIST-gil, Ulsan, Korea

${ }^{30}$ Earthquake Research Institute, University of Tokyo, Bunkyo-ku, Tokyo, Japan

${ }^{31}$ Graduate School of Information Sciences, Hiroshima City University, Hiroshima, Hiroshima, Japan

32 Institute of Particle and Nuclear Studies, KEK, Tsukuba, Ibaraki, Japan

${ }^{33}$ Graduate School of Science and Engineering, Tokyo Institute of Technology, Meguro, Tokyo, Japan

${ }^{34}$ Department of Research Planning and Promotion, Quantum Medical Science Directorate, National Institutes for Quantum and Radiological Science and Technology, Chiba, Chiba, Japan

${ }^{35}$ CEICO, Institute of Physics, Czech Academy of Sciences, Prague, Czech Republic

${ }^{36}$ Department of Physics and Institute for the Early Universe, Ewha Womans University, Seodaaemun-gu, Seoul, Korea

** Deceased 